\title{
Fuzzy Stabilization for Nonlinear Discrete Ship Steering Stochastic Systems Subject to State Variance and Passivity Constraints
}

\author{
Wen-Jer Chang, Bo-Jyun Huang, and Po-Hsun Chen \\ Department of Marine Engineering, National Taiwan Ocean University, Keelung 202, Taiwan \\ Correspondence should be addressed to Wen-Jer Chang; wjchang@mail.ntou.edu.tw
}

Received 19 October 2013; Accepted 21 November 2013; Published 9 January 2014

Academic Editor: Shuping He

Copyright (c) 2014 Wen-Jer Chang et al. This is an open access article distributed under the Creative Commons Attribution License, which permits unrestricted use, distribution, and reproduction in any medium, provided the original work is properly cited.

For nonlinear discrete-time stochastic systems, a fuzzy controller design methodology is developed in this paper subject to state variance constraint and passivity constraint. According to fuzzy model based control technique, the nonlinear discrete-time stochastic systems considered in this paper are represented by the discrete-time Takagi-Sugeno fuzzy models with multiplicative noise. Employing Lyapunov stability theory, upper bound covariance control theory, and passivity theory, some sufficient conditions are derived to find parallel distributed compensation based fuzzy controllers. In order to solve these sufficient conditions, an iterative linear matrix inequality algorithm is applied based on the linear matrix inequality technique. Finally, the fuzzy stabilization problem for nonlinear discrete ship steering stochastic systems is investigated in the numerical example to illustrate the feasibility and validity of proposed fuzzy controller design method.

\section{Introduction}

The nonlinear control systems have gained more and more research attention, and lots of results have been published [1-5]. When analyzing and designing nonlinear dynamical systems, there are a wide range of nonlinear analysis tools, among which the most common and wildly used is linearization because of the powerful tools we know for linear systems. On the other hand, due to the wide appearance of the stochastic phenomena in almost every aspect of our daily life, stochastic systems which have found successful applications in many branches of science and engineering practice have stirred quite a lot of research interests during the past few decades; see $[1,2,6,7]$ and the references therein. Therefore, the control problems for nonlinear stochastic systems have been studied extensively so as to meet everincreasing demand toward systems with both nonlinearities and stochasticity. As far as nonlinear systems are concerned, the multiple performance constrained control problem has gained some research interests, for example, mixed $\mathrm{H}_{2} / \mathrm{H}_{\infty}$ control [8-10]. However, the $H_{2} / H_{\infty}$ controllers minimize a linear quadratic performance index without guaranteeing the variance constraints with respect to individual system states. In order to deal with the individual state variance constrained control problem, the extending approach of covariance control theory $[1,2,11,12]$ is investigated in this paper subject to passivity performance constraint.

The performance constraints considered in this paper include individual state variance constraint and passivity performance constraint. The covariance control theory $[1,2$, 11,12 ] has provided a more direct methodology for achieving the individual variance constraints than the Linear Quadratic Gaussian (LQG) control theory. It has been shown that the covariance control approach is capable of solving multiobjective design problems, which has found applications in dealing with transient responses, round off errors in digital control, residence time/probability in aiming control problems, and stability, robustness in the presence of parameter perturbations [13]. In fact, this type of control that provides the ability to assign the second moment of the system state offers many advantages, such as higher performance and improved robustness. Extending the covariancecontrol theory, the individual state variance constrainedcontrol problem for the nonlinear stochastic systems is alsosuccessfully investigated 
based on Takagi-Sugeno (T-S) fuzzy models [14, 15]. However, the difficulty of covariance control methods [1, 2, 11-15] is that the designers should assign a suitable state covariance matrix to achieve the Lyapunov equation conditions. In order to improve the covariance control approach, this paper tries to derive novel sufficient conditions that can be solved by Linear Matrix Inequality (LMI) technique.

In addition to individual state variance constraint, the passivity constraint is also considered in this paper. In recent years, the theory of passive systems,c which plays an important role in system and control areas, has been attracting a great deal of research interests and many results have been reported so far; see [16-25]. In [16, 17], an LMI method was used to design the state feedback controller ensuring both the asymptotic stability and strictlyquadratic passivity. In [18], the passive control problem hasbeen solved for time-delay systems. For nonlinear controlsystems with multiplicative noises, the passivity constrained control was studied in $[21,22]$ via T-S fuzzy models. The passive theory provides us a useful tool for the analysis of systems with multiple performance criteria. However, few literatures have been concerned with the multiobjective design problem for nonlinear stochastic systems. So far, the variance-constrained controller design problem with passivity constraint has not yet been studied adequately for nonlinear discrete-time stochastic systems.

It is worth mentioning that fuzzy control of nonlinear discrete-time stochastic systems subject to individual state variance constraint and passivity constraint is still a challenging problem for the control engineers. Thus, the motivation of this paper is to investigate the multiple constrained fuzzy controller design methodology for nonlinear discrete-time stochastic systems via T-S fuzzy models with multiplicative noises. T-S fuzzy model is constructed by a set of local linear subsystems. Then, a model-based fuzzy controller can be developed to stabilize this T-S fuzzy model $[10,14,15,21,22$, 26-28]. The advantage of controller synthesis for such a fuzzy model is that the linear control methods can be used. Based on the Parallel Distributed Compensation (PDC) technique $[10,14,15,21,22]$, some approaches have been developed to design the fuzzy controllers for manynonlinear systems via T-S fuzzy models. In addition, fuzzycontrol approach was also successfully employed to design controllers for dynamic systems with Markov jumps in $[29,30]$. Applying the PDC based fuzzy control, the sufficient conditions derived in this paper are mostly Bilinear Matrix Inequality (BMI) problems which are difficult to solve by numerically convex optimization technique. For this reason, an iterative manner, which is called Iterative Linear Matrix Inequality (ILMI) algorithm [31, 32], is applied in this paper to find suitable fuzzy control gains.

By employing PDC technique, the first contribution of this paper is to develop a fuzzy control approach for nonlinear discrete-time stochastic systems such that individual state variance constraint and passivity constraint are all satisfied. For the practical nonlinear stochastic systems represented by the T-S fuzzy models with multiplicative noises, the second contribution of this paper is to develop an ILMI algorithm to solve the derived BMI sufficient conditions that achieve individual state variance constraint and passivity constraint, simultaneously. In order to illustrate the applicability and efficiency of proposed fuzzy controller design approach, a nonlinear discrete ship steering stochastic system is considered in this paper. Based on the T-S fuzzy modeling technique, the fuzzy controller design methods for the nonlinear discrete ship steering system have been developed in $[33,34]$. Considering the nonlinear discrete ship steering system in $[33,34]$, a fuzzy controller design methodology is investigated in the numerical example to achieve the above multiple performance constraints.

\section{Performance Constraint Descriptions and Problem Statements}

Consider a nonlinear discrete ship steering stochastic system, which is constructed by a discrete-time T-S fuzzy model with multiplicative noise as follows.

Rule $i$ :

$$
\begin{aligned}
& \text { IF } z_{1}(k) \text { is } M_{i 1} \text { and } \cdots \text { and } z_{q}(k) \text { is } M_{i q} \\
& \text { then } x(k+1) \\
& =\left[\mathbf{A}_{i}+\sum_{e=1}^{m} \mathbf{N}_{e i} v_{e i}(k)\right] x(k)+\mathbf{B}_{i} u(k)+\mathbf{D}_{i} w(k) \\
& \qquad y(k)=\mathbf{C}_{i} x(k)+\mathbf{H}_{i} w(k),
\end{aligned}
$$

where $z_{1}(k), \ldots, z_{q}(k)$ are the premise variables, $M_{i q}$ is the fuzzy set, $q$ is the premise variable number, $r$ is the number of fuzzy rules, $x(k) \in \mathfrak{R}^{n_{x}}$ is the state vector, $u(k) \in \mathfrak{R}^{n_{u}}$ is the input vector, $y(k) \in \Re^{n_{y}}$ is the output vector, and the processes $w(k) \in \mathfrak{R}^{n_{w}}$ and $v_{e i}(t) \in \Re$ are mutually independent zero-mean Gaussian white noise with intensities $\mathbf{W}(\mathbf{W}>0)$ and 1 , respectively. It is assumed that $E\{w(t)\}=0, E\left\{v_{e i}(t)\right\}=0, E\{x(t) w(t)\}=E\{x(t)\} E\{w(t)\}=0$, $E\left\{x(t) v_{e i}(t)\right\}=E\{x(t)\} E\left\{v_{e i}(t)\right\}=0$, and $E\left\{w(t) v_{e i}(t)\right\}=$ $E\{w(t)\} E\left\{v_{e i}(t)\right\}=0$. The matrices $\mathbf{A}_{i} \in \mathfrak{R}^{n_{x} \times n_{x}}, \mathbf{B}_{i} \in \mathfrak{R}^{n_{x} \times n_{u}}$, $\mathbf{D}_{i} \in \mathfrak{R}^{n_{x} \times n_{w}}, \mathbf{N}_{e i} \in \mathfrak{R}^{n_{x} \times n_{x}}, \mathbf{C}_{i} \in \mathfrak{R}^{n_{y} \times n_{x}}$, and $\mathbf{H}_{i} \in \mathfrak{R}^{n_{y} \times n_{w}}$ are constant.

Given the pair $(x(k), u(k))$, the overall fuzzy model can be described as follows:

$$
\begin{aligned}
& x(k+1)=\sum_{i=1}^{r} h_{i}(z(k)) \\
& \times\left\{\left[\mathbf{A}_{i}+\sum_{e=1}^{m} \mathbf{N}_{e i} v_{e i}(k)\right]\right. \\
& \left.\times x(k)+\mathbf{B}_{i} u(k)+\mathbf{D}_{i} w(k)\right\}, \\
& y(k)=\sum_{i=1}^{r} h_{i}(z(k))\left\{\mathbf{C}_{i} x(k)+\mathbf{H}_{i} w(k)\right\},
\end{aligned}
$$


where $h_{i}(z(k))=\omega_{i}(z(k)) / \sum_{i=1}^{r} \omega_{i}(z(k)), \omega_{i}(z(k))=\prod_{j=1}^{q} M_{i j}$ $\left(z_{j}(k)\right), h_{i}(z(k)) \geq 0$, and $\sum_{i=1}^{r} h_{i}(z(k))=1$.

Applying PDC technique, the fuzzy controller is designed to share the same IF part of the T-S fuzzy model (1a) and (1b). The corresponding fuzzy controller can be represented as follows.

Rule $i$ :

$$
\begin{aligned}
& \text { IF } z_{1}(k) \text { is } M_{i 1} \text { and } \ldots \text { and } z_{q}(k) \text { is } M_{i q} \\
& \text { THEN } u(k)=\mathbf{F}_{i} x(k)
\end{aligned}
$$

or

$$
u(k)=\sum_{i=1}^{r} h_{i}(z(k))\left\{\mathbf{F}_{i} x(k)\right\} .
$$

Substituting (4) into (2a), the closed-loop T-S fuzzy model can be obtained as follows:

$$
\begin{aligned}
x(k+1)=\sum_{i=1}^{r} \sum_{j=1}^{r} h_{i}(z(k)) h_{j}(z(k)) \\
\times\left[\left(\mathbf{A}_{i}+\sum_{e=1}^{m} \mathbf{N}_{e i} v_{e i}(k)+\mathbf{B}_{i} \mathbf{F}_{j}\right)\right. \\
\left.\times x(k)+\mathbf{D}_{i} w(k)\right] .
\end{aligned}
$$

The first performance constraint considered in this paper is the passivity constraint. Via different definitions of supply function of passivity theory, the systems can be stabilized for achieving different types of attenuation performance with requirement energy. In general, the strictly input passive type constraint is usually used to constrain the disturbance effect and to study the stability of fuzzy systems. The strictly input passive constraint can be introduced in the following definition.

Definition 1 (see [19]). The system (5) with external disturbance $w(k)$ and output $y(k)$ is said to be strictly input passive if the following inequality is achieved:

$$
E\left\{2 \sum_{k=0}^{k_{q}} y^{T}(k) w(k)\right\}>E\left\{\sum_{k=0}^{k_{q}} \gamma w^{T}(k) w(k)\right\}
$$

for all $\gamma \geq 0$ and $k_{q}$ is a positive integer that denotes terminal sampled time of control.

Considering each subsystem of the T-S fuzzy model (5), the steady state covariance matrix of the state vector $x(t)$ has the following form:

$$
\mathbf{X}_{i}=\mathbf{X}_{i}^{T}>0
$$

where $\mathbf{X}_{i}=\lim _{k \rightarrow \infty} E\left[x(k) x^{T}(k)\right]$ and $\mathbf{X}_{i}$ is the unique solution of the following Lyapunov equation for each rule [35]:

$$
\begin{array}{r}
\left(\mathbf{A}_{i}+\mathbf{B}_{i} \mathbf{F}_{i}\right) \mathbf{X}_{i}\left(\mathbf{A}_{i}+\mathbf{B}_{i} \mathbf{F}_{i}\right)^{T}-\mathbf{X}_{i} \\
+\mathbf{D}_{i} \mathbf{W} \mathbf{D}_{i}^{T}+\sum_{e=1}^{m} \mathbf{N}_{e i} \mathbf{X}_{i} \mathbf{N}_{e i}^{T}=0 \\
i=1,2, \ldots, r .
\end{array}
$$

The second performance constraint considered in this paper is the state variance constraint that is defined as follows:

$$
\left[\mathbf{X}_{i}\right]_{k k} \leq \sigma_{k}^{2},
$$

where $[\cdot]_{k k}$ denotes the $k$ th diagonal element of matrix $[\cdot]$ and $\sigma_{k}, k=1,2, \ldots, n_{x}$, denote the root-mean-squared constraints for the variance of system states.

The following lemma is useful for the derivations of the subsequent theorem that provides sufficient conditions to achieve the stability, passivity constraint (6), and individual state variance constraint (9) for the closed-loop system (5).

Lemma 2 (see [36]). Given constant matrices $\mathbf{E}_{1}, \mathbf{E}_{2}$, and $\mathbf{E}_{3}$, where $\mathbf{E}_{1}=\mathbf{E}_{1}^{T}$ and $\mathbf{E}_{2}=\mathbf{E}_{2}^{T}>0$, then $\mathbf{E}_{1}+\mathbf{E}_{3} \mathbf{E}_{2}^{-1} \mathbf{E}_{3}^{T}<0$ if and only if

$$
\left[\begin{array}{cc}
\mathbf{E}_{1} & \mathbf{E}_{3}^{T} \\
\mathbf{E}_{3} & -\mathbf{E}_{2}
\end{array}\right]<0 \text { or equivalently }\left[\begin{array}{cc}
-\mathbf{E}_{2} & \mathbf{E}_{3} \\
\mathbf{E}_{3}^{T} & \mathbf{E}_{1}
\end{array}\right]<0 .
$$

The purpose of this paper is to find feedback gains $\mathbf{F}_{i}$ that can be used to construct the PDC-based fuzzy controller (4) such that the passivity constraint (6) and individual state variance constraint (9) are all satisfied, simultaneously.

\section{Condition Derivation for Achieving Multiple Performance Constraints}

In this section, the sufficient conditions are derived to guarantee the stability, individual variance constraint, and passivity constraint of closed-loop T-S fuzzy model with multiplicative noises. These sufficient conditions are derived based on the Lyapunov theory and passivity theory. Improving the covariance control theory, the sufficient conditions are derived in the following theorem by assigning a common upper bound matrix of the state covariance matrices for all fuzzy rules.

Theorem 3. If there exist positive definite matrices $\overline{\mathbf{X}}>0$, $\mathbf{P}>0$, feedback gains $\mathbf{F}_{i}$, and dissipative rate $\gamma$ satisfying the following sufficient conditions, then the closed-loop system (5) is asymptotically stable, strictly input passive and $\left[\mathbf{X}_{i}\right]_{k k} \leq \sigma_{k}^{2}$ :

$$
\begin{gathered}
{\left[\begin{array}{cc}
\mathbf{Q}_{i j} & \Gamma_{i}^{T} \\
\Gamma_{i} & \Omega
\end{array}\right]<0,} \\
\overline{\mathbf{X}}<\operatorname{diag}\left(\sigma_{1}^{2}, \ldots, \sigma_{n_{x}}^{2}\right),
\end{gathered}
$$

where 


$$
\begin{aligned}
& \mathbf{Q}_{i j}=\left[\begin{array}{ccccc}
-\overline{\mathbf{X}}+\mathbf{D}_{i} \mathbf{W} \mathbf{D}_{i}^{T}+\sum_{e=1}^{m} \mathbf{N}_{e i} \overline{\mathbf{X}} \mathbf{N}_{e i}^{T} & \mathbf{G}_{i j} & 0 & 0 & 0 \\
\mathbf{G}_{i j}^{T} & -\mathbf{P} & -\mathbf{C}_{i}^{T} & 0 & \mathbf{G}_{i j}^{T} \\
0 & -\mathbf{C}_{i} & \mathbf{D}_{i}^{T} \mathbf{P} \mathbf{D}_{i}+\underset{\gamma}{ } \mathbf{I}-\mathbf{H}_{i}-\mathbf{H}_{i}^{T} & \mathbf{D}_{i}^{T} \mathbf{P} & 0 \\
0 & 0 & \mathbf{P D}_{i} & -\mathbf{I} & 0 \\
0 & \mathbf{G}_{i j} & 0 & 0 & -\mathbf{I}
\end{array}\right], \\
& \Gamma_{i}=\left[\begin{array}{ccccc}
0 & \mathbf{N}_{1 i} & 0 & 0 & 0 \\
\vdots & \vdots & \vdots & \vdots & \vdots \\
0 & \mathbf{N}_{m i} & 0 & 0 & 0
\end{array}\right] \text {, } \\
& \Omega=\operatorname{diag}\left(-\frac{\overline{\mathbf{x}}}{m}, \ldots,-\frac{\overline{\mathbf{X}}}{m}\right) .
\end{aligned}
$$

In addition, $\mathbf{G}_{i j}=\mathbf{A}_{i}+\mathbf{B}_{i} \mathbf{F}_{j}, \mathbf{P}=\overline{\mathbf{X}}^{-1}$, and $\operatorname{diag}\left(\sigma_{1}^{2}, \ldots, \sigma_{n_{x}}^{2}\right)$ denotes a diagonal matrix with the diagonal elements $\sigma_{1}^{2}, \sigma_{2}^{2}, \ldots$, and $\sigma_{n_{x}}^{2}$.

Proof. In order to analyze the stability of the closed-loop T-S fuzzy system (5), a Lyapunov function is chosen as
$V(x(k))=x^{T}(k) \mathbf{P} x(k)$, where $\mathbf{P}=\mathbf{P}^{T}>0$. The expected value of differential of the Lyapunov function $V(x(k))$ along the trajectories of (5) is given as follows:

$$
\begin{aligned}
E\{ & \Delta V(x(k))\} \\
=E\left\{x^{T}(k+1) \mathbf{P} x(k+1)-x^{T}(k) \mathbf{P} x(k)\right\} & \left\{\sum_{i=1}^{r} \sum_{j=1}^{r} \sum_{k=1}^{r} \sum_{l=1}^{r} h_{i}(z(k)) h_{j}(z(k)) h_{k}(z(k)) h_{l}(z(k))\right. \\
= & \left\{\left[\left(\mathbf{A}_{i}+\sum_{e=1}^{m} \mathbf{N}_{e i} v_{e i}(k)+\mathbf{B}_{i} \mathbf{F}_{j}\right) x(k)+\mathbf{D}_{i} w(k)\right]^{T} \mathbf{P}\right. \\
& \left.\left.\times\left[\left(\mathbf{A}_{k}+\sum_{g=1}^{m} \mathbf{N}_{g i} v_{g i}(k)+\mathbf{B}_{k} \mathbf{F}_{l}\right) x(k)+\mathbf{D}_{k} w(k)\right]-x^{T}(k) \mathbf{P} x(k)\right\}\right\} \\
\leq E & \left\{\sum_{i=1}^{r} \sum_{j=1}^{r} h_{i}(z(k)) h_{j}(z(k))\right. \\
\times & \left\{\left[\left(\mathbf{A}_{i}+\sum_{e=1}^{m} \mathbf{N}_{e i} v_{e i}(k)+\mathbf{B}_{i} \mathbf{F}_{j}\right) x(k)+\mathbf{D}_{i} w(k)\right]^{T} \mathbf{P}\right.
\end{aligned}
$$




$$
\begin{aligned}
& \left.\left.\times\left[\left(\mathbf{A}_{i}+\sum_{g=1}^{m} \mathbf{N}_{g i} v_{g i}(k)+\mathbf{B}_{i} \mathbf{F}_{j}\right) x(k)+\mathbf{D}_{i} w(k)\right]-x^{T}(k) \mathbf{P} x(k)\right\}\right\} \\
& =E\left\{\sum_{i=1}^{r} \sum_{j=1}^{r} h_{i}(z(k)) h_{j}(z(k))\right. \\
& \times\left\{x^{T}(k)\left(\mathbf{A}_{i}+\mathbf{B}_{i} \mathbf{F}_{j}\right)^{T} \mathbf{P}\left(\mathbf{A}_{i}+\mathbf{B}_{i} \mathbf{F}_{j}\right) x(k)\right. \\
& -x^{T}(k) \mathbf{P} x(k)+x^{T}(k)\left(\mathbf{A}_{i}+\mathbf{B}_{i} \mathbf{F}_{j}\right)^{T} \mathbf{P} \mathbf{D}_{i} w(k) \\
& +w^{T}(k) \mathbf{D}_{i}^{T} \mathbf{P}\left(\mathbf{A}_{i}+\mathbf{B}_{i} \mathbf{F}_{j}\right) x(k) \\
& +w^{T}(k) \mathbf{D}_{i}^{T} \mathbf{P} \mathbf{D}_{i} w(k)+\sum_{g=1}^{m} x^{T}(k)\left(\mathbf{A}_{i}+\mathbf{B}_{i} \mathbf{F}_{j}\right)^{T} \mathbf{P N}_{g i} v_{g i}(k) x(k) \\
& +\sum_{e=1}^{m} x^{T}(k) v_{e i}^{T}(k) \mathbf{N}_{e i}^{T} \mathbf{P}\left(\mathbf{A}_{i}+\mathbf{B}_{i} \mathbf{F}_{j}\right) x(k)+\sum_{g=1}^{m} w^{T}(k) \mathbf{D}_{i}^{T} \mathbf{P N}_{g i} v_{g i}(k) x(k) \\
& \left.\left.+\sum_{e=1}^{m} x^{T}(k) v_{e i}^{T}(k) \mathbf{N}_{e i}^{T} \mathbf{P} \mathbf{D}_{i} w(k)+\sum_{e=1}^{m} \sum_{g=1}^{m} x^{T}(k) v_{e i}^{T}(k) \mathbf{N}_{e i}^{T} \mathbf{P N}_{g i} v_{g i}(k) x(k)\right\}\right\} \\
& =E\left\{\sum_{i=1}^{r} \sum_{j=1}^{r} h_{i}(z(k)) h_{j}(z(k))\right. \\
& \times\left\{x^{T}(k)\left(\mathbf{A}_{i}+\mathbf{B}_{i} \mathbf{F}_{j}\right)^{T} \mathbf{P}\left(\mathbf{A}_{i}+\mathbf{B}_{i} \mathbf{F}_{j}\right) x(k)\right. \\
& -x^{T}(k) \mathbf{P} x(k)+x^{T}(k)\left(\mathbf{A}_{i}+\mathbf{B}_{i} \mathbf{F}_{j}\right)^{T} \mathbf{P} \mathbf{D}_{i} w(k) \\
& +w^{T}(k) \mathbf{D}_{i}^{T} \mathbf{P}\left(\mathbf{A}_{i}+\mathbf{B}_{i} \mathbf{F}_{j}\right) x(k) \\
& \left.\left.+w^{T}(k) \mathbf{D}_{i}^{T} \mathbf{P D}_{i} w(k)+\sum_{e=1}^{m} \sum_{g=1}^{m} x^{T}(k) \mathbf{N}_{e i}^{T} \mathbf{P} \mathbf{N}_{g i} x(k)\right\}\right\} \\
& \leq E\left\{\sum_{i=1}^{r} \sum_{j=1}^{r} h_{i}(z(k)) h_{j}(z(k))\right. \\
& \times\left\{x^{T}(k)\left(\mathbf{A}_{i}+\mathbf{B}_{i} \mathbf{F}_{j}\right)^{T} \mathbf{P}\left(\mathbf{A}_{i}+\mathbf{B}_{i} \mathbf{F}_{j}\right) x(k)\right. \\
& -x^{T}(k) \mathbf{P} x(k)+x^{T}(k)\left(\mathbf{A}_{i}+\mathbf{B}_{i} \mathbf{F}_{j}\right)^{T}\left(\mathbf{A}_{i}+\mathbf{B}_{i} \mathbf{F}_{j}\right) x(k) \\
& +w^{T}(k) \mathbf{D}_{i}^{T} \mathbf{P P D}_{i} w(k) \\
& \left.\left.+w^{T}(k) \mathbf{D}_{i}^{T} \mathbf{P} \mathbf{D}_{i} w(k)+m \sum_{e=1}^{m} x^{T}(k) \mathbf{N}_{e i}^{T} \mathbf{P N}_{e i} x(k)\right\}\right\}
\end{aligned}
$$




$$
\begin{aligned}
= & E\left\{\sum_{i=1}^{r} \sum_{j=1}^{r} h_{i}(z(k)) h_{j}(z(k))\right. \\
& \times\left\{\left[\begin{array}{c}
x(k) \\
w(k)
\end{array}\right]^{T}\right. \\
& \left.\times\left[\begin{array}{c}
\left(\mathbf{A}_{i}+\mathbf{B}_{i} \mathbf{F}_{j}\right)^{T} \mathbf{P}\left(\mathbf{A}_{i}+\mathbf{B}_{i} \mathbf{F}_{j}\right)-\mathbf{P}+\left(\mathbf{A}_{i}+\mathbf{B}_{i} \mathbf{F}_{j}\right)^{T}\left(\mathbf{A}_{i}+\mathbf{B}_{i} \mathbf{F}_{j}\right)+m \sum_{e=1}^{m} \mathbf{N}_{e i}^{T} \mathbf{P N} \mathbf{N}_{e i} \\
0
\end{array}\right] \mathbf{D}_{i}^{T} \mathbf{P P D}_{i}+\mathbf{D}_{i}^{T} \mathbf{P D}_{i}\right] \\
& \left.\left.\times\left[\begin{array}{c}
x(k) \\
w(k)
\end{array}\right]\right\}\right\} .
\end{aligned}
$$

It is obvious that if condition (11) is satisfied, one has

$$
\left[\begin{array}{cc}
-\overline{\mathbf{X}}+\mathbf{D}_{i} \mathbf{W} \mathbf{D}_{i}^{T}+\sum_{e=1}^{m} \mathbf{N}_{e i} \overline{\mathbf{X}} \mathbf{N}_{e i}^{T} & \mathbf{A}_{i}+\mathbf{B}_{i} \mathbf{F}_{i} \\
\left(\mathbf{A}_{i}+\mathbf{B}_{i} \mathbf{F}_{i}\right)^{T} & -\mathbf{P}
\end{array}\right]<0 .
$$

Using Lemma 2, one can obtain the following inequality by assigning $\overline{\mathbf{X}}=\mathbf{P}^{-1}$ :

$$
\left(\mathbf{A}_{i}+\mathbf{B}_{i} \mathbf{F}_{i}\right) \overline{\mathbf{X}}\left(\mathbf{A}_{i}+\mathbf{B}_{i} \mathbf{F}_{i}\right)^{T}-\overline{\mathbf{X}}+\mathbf{D}_{i} \mathbf{W} \mathbf{D}_{i}^{T}+\sum_{e=1}^{m} \mathbf{N}_{e i} \overline{\mathbf{X}} \mathbf{N}_{e i}^{T}<0
$$

Subtracting (7) from (16), one has

$$
\begin{aligned}
\left(\mathbf{A}_{i}+\right. & \left.\mathbf{B}_{i} \mathbf{F}_{i}\right)\left(\overline{\mathbf{X}}-\mathbf{X}_{i}\right)\left(\mathbf{A}_{i}+\mathbf{B}_{i} \mathbf{F}_{i}\right)^{T}-\left(\overline{\mathbf{X}}-\mathbf{X}_{i}\right) \\
& +\sum_{e=1}^{m} \mathbf{N}_{e i}\left(\overline{\mathbf{X}}-\mathbf{X}_{i}\right) \mathbf{N}_{e i}^{T}<0
\end{aligned}
$$

Because the conditions (16) is satisfied, one can obtain that the closed-loop system (5) is stable and the closed-loop system matrix $\left(\mathbf{A}_{i}+\mathbf{B}_{i} \mathbf{F}_{i}\right)$ is stable. In this case, it can be concluded that $\overline{\mathbf{X}}-\mathbf{X}_{i} \geq 0$ via the inequality (17). From condition (12) and $\overline{\mathbf{X}} \geq \mathbf{X}_{i}$, one has

$$
\left[\mathbf{X}_{i}\right]_{k k} \leq[\overline{\mathbf{X}}]_{k k} \leq \sigma_{k}^{2}
$$

Thus, the individual state variance constraint (9) can be achieved.
In order to achieve the passivity constraint defined in Definition 1, let us define a performance function such as

$$
\begin{aligned}
& E\left\{\sum_{k=0}^{k_{q}}\left(\gamma w^{T}(k) w(k)-2 y^{T}(k) w(k)\right)\right\} \\
& \leq E\left\{\sum_{k=0}^{k_{q}}\left(\gamma w^{T}(k) w(k)-2 y^{T}(k) w(k)\right)+V(x(k))\right\} \\
& =E\left\{\sum_{k=0}^{k_{q}}\left(\gamma w^{T}(k) w(k)-2 y^{T}(k) w(k)\right)\right. \\
& \left.+\sum_{k=0}^{k_{q}} \Delta V(x(k))\right\} \\
& \triangleq E\left\{\sum_{k=0}^{k_{q}}\left(\gamma w^{T}(k) w(k)-2 y^{T}(k) w(k)+\Delta V(x(k))\right)\right\} \\
& \triangleq E\{K(x, w, k)\},
\end{aligned}
$$

where

$$
\begin{aligned}
& K(x, w, k) \\
& \triangleq \sum_{k=0}^{k_{q}}\left(\gamma w^{T}(k) w(k)-2 y^{T}(k) w(k)+\Delta V(x(k))\right) .
\end{aligned}
$$

Using inequality (14), one has

$$
\begin{aligned}
E & \{K(x, w, k)\} \\
& =E\left\{\sum_{i=1}^{r} \sum_{j=1}^{r} h_{i}(z(k)) h_{j}(z(k))\left[\begin{array}{c}
x(k) \\
w(k)
\end{array}\right]^{T}\right.
\end{aligned}
$$




$$
\begin{aligned}
& \times\left[\begin{array}{cc}
\mathbf{G}_{i j}^{T} \mathbf{P} \mathbf{G}_{i j}-\mathbf{P}+\mathbf{G}_{i j}^{T} \mathbf{G}_{i j}+m \sum_{e=1}^{m} \mathbf{N}_{e i}^{T} \mathbf{P} \mathbf{N}_{e i} & -\mathbf{C}_{i}^{T} \\
-\mathbf{C}_{i} & \mathbf{D}_{i}^{T} \mathbf{P P D}_{i}+\mathbf{D}_{i}^{T} \mathbf{P D}_{i}+\gamma \mathbf{I}-\mathbf{H}_{i}-\mathbf{H}_{i}^{T}
\end{array}\right] \\
& \left.\times\left[\begin{array}{c}
x(k) \\
w(k)
\end{array}\right]\right\}
\end{aligned}
$$

Due to $\mathbf{D}_{i} \mathbf{W} \mathbf{D}_{i}^{T} \geq 0$ and $\sum_{e=1}^{m} \mathbf{N}_{e i} \overline{\mathbf{X}} \mathbf{N}_{e i}^{T}>0$, it is obvious that condition (11) implies

$$
\left[\begin{array}{cccccccc}
-\overline{\mathbf{X}} & \mathbf{G}_{i j} & 0 & 0 & 0 & 0 & \cdots & 0 \\
\mathbf{G}_{i j}^{T} & -\mathbf{P} & -\mathbf{C}_{i}^{T} & 0 & \mathbf{G}_{i j}^{T} & \mathbf{N}_{1 i}^{T} & \cdots & \mathbf{N}_{m i}^{T} \\
0 & -\mathbf{C}_{i} & \mathbf{D}_{i}^{T} \mathbf{P D}_{i}+\gamma \mathbf{I}-\mathbf{H}_{i}-\mathbf{H}_{i}^{T} & \mathbf{D}_{i}^{T} \mathbf{P} & 0 & 0 & \cdots & 0 \\
0 & 0 & \mathbf{P D}_{i} & -\mathbf{I} & 0 & 0 & \cdots & 0 \\
0 & \mathbf{G}_{i j} & 0 & 0 & -\mathbf{I} & 0 & \cdots & 0 \\
0 & \mathbf{N}_{1 i} & 0 & 0 & 0 & -\frac{\mathbf{X}}{m} & \cdots & 0 \\
\vdots & \vdots & \vdots & \vdots & \vdots & \vdots & \ddots & \vdots \\
0 & \mathbf{N}_{m i} & 0 & 0 & 0 & 0 & \cdots & -\frac{\mathbf{X}}{m}
\end{array}\right]<0
$$

Using inequality (22) and Lemma 2, one has

$$
\left[\begin{array}{cc}
\mathbf{G}_{i j}^{T} \mathbf{P G}_{i j}-\mathbf{P}+\mathbf{G}_{i j}^{T} \mathbf{G}_{i j}+m \sum_{e=1}^{m} \mathbf{N}_{e i}^{T} \mathbf{P} \mathbf{N}_{e i} & -\mathbf{C}_{i}^{T} \\
-\mathbf{C}_{i} & \mathbf{D}_{i}^{T} \mathbf{P} \mathbf{P} \mathbf{D}_{i}+\mathbf{D}_{i}^{T} \mathbf{P D}_{i}+\gamma \mathbf{I}-\mathbf{H}_{i}-\mathbf{H}_{i}^{T}
\end{array}\right]<0 .
$$

If condition (23) holds, then one can obtain $E\{K(x, w, k)\}<$ 0 . From (20), the inequality $E\{K(x, w, k)\}<0$ implies

$$
E\left\{2 \sum_{k=0}^{k_{q}} y^{T}(k) w(k)\right\}>E\left\{\sum_{k=0}^{k_{q}} \gamma w^{T}(k) w(k)\right\} .
$$

From Definition 1, it can be thus concluded that if condition (11) is satisfied, then the closed-loop system (5) is strictly input passive.

Remark 4. In conditions (11) and (12) of Theorem 3, the variables $\overline{\mathbf{X}}, \mathbf{P}$, and $\mathbf{K}_{i}$ are needed to be found. With increasing the number of fuzzy rules, the number of inequality conditions is increasing such that the difficulty of finding the desired variables is also increased. Thus, the computational complexity of the proposed approach will be increased when the number of fuzzy rules is arisen. For reducing the computational complexity, the nonlinear system is recommended to be modeled by T-S fuzzy model as less fuzzy rules as possible.
In Theorem 3, the conditions simultaneously include variables $\mathbf{P}$ and $\overline{\mathbf{X}}$ (i.e., $\mathbf{P}^{-1}$ ) such that the conditions are not a strictly LMI problem. In order to apply the LMI technique to solve conditions of Theorem 3, the ILMI algorithm is employed to solve the nonlinear minimization problem of (11) and (12). The ILMI algorithm has been applied to solve the fuzzy controller design problem for the affine T-S fuzzy systems [31, 32]. In this paper, a modified ILMI algorithm is developed to solve the proposed nonlinear matrix inequality conditions subject to multiple performance constraints. According to conditions (11) and (12), the modified ILMI algorithm is described as follows.

\section{Algorithm 5 (modified ILMI algorithm)}

Step 1. Find the feasible solutions $\mathbf{P}^{0}$ and $\overline{\mathbf{X}}^{0}$ for satisfying (11)-(12). Then the initial variables can be obtained as $\left\{\mathbf{P}^{0}, \overline{\mathbf{X}}^{0}\right\}$. Set $t=1$ and go to Step 2 . 


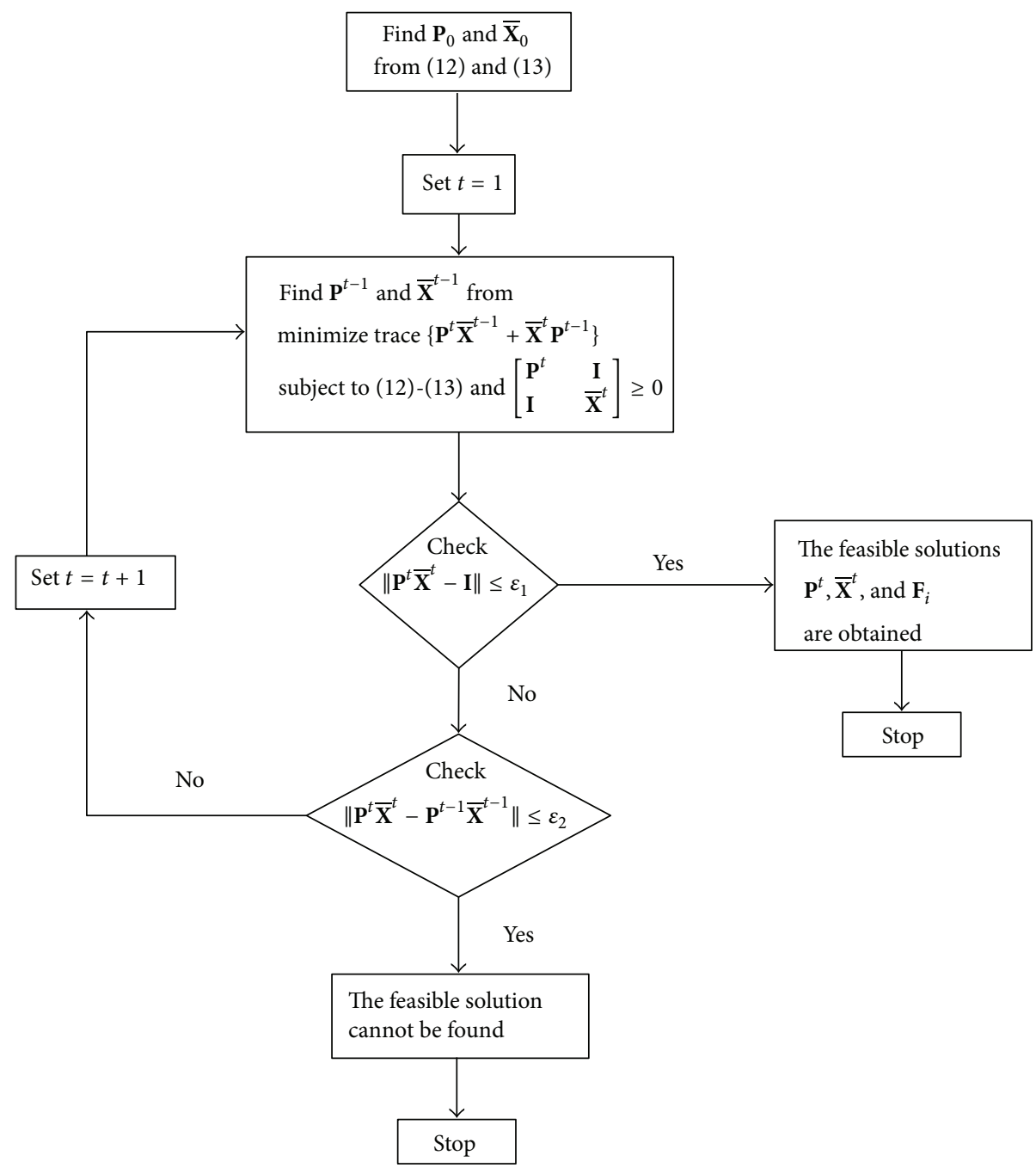

FIGURE 1: Flowchart of ILMI algorithm for finding control gains.

Step 2. Use obtained variables $\left\{\mathbf{P}^{t-1}, \overline{\mathbf{X}}^{t-1}\right\}$ to solve the following LMI problem for desired variables $\left\{\mathbf{P}^{t}, \overline{\mathbf{X}}^{t}\right\}$ :

$$
\begin{aligned}
& \text { minimize } \operatorname{trace}\left\{\mathbf{P}^{t} \overline{\mathbf{X}}^{t-1}+\overline{\mathbf{X}}^{t} \mathbf{P}^{t-1}\right\} \\
& \text { subject to } \quad(11)-(12), \quad\left[\begin{array}{cc}
\mathbf{P}^{\mathrm{t}} & \mathbf{I} \\
\mathbf{I} & \overline{\mathbf{X}}^{\mathrm{t}}
\end{array}\right] \geq 0 .
\end{aligned}
$$

Step 3. If condition (26) is satisfied, then the feasible solutions $\mathbf{P}^{t}, \overline{\mathbf{X}}^{t}$, and $\mathbf{F}_{i}$ are obtained and the algorithm can be stopped. Otherwise, go to Step 4:

$$
\left\|\mathbf{P}^{t} \overline{\mathbf{X}}^{t}-\mathbf{I}\right\|<\varepsilon_{1}
$$

where $\varepsilon_{1}>0$ is a small scalar.

Step 4. If condition (27) is satisfied, then this algorithm cannot find feasible solutions and one can quit the algorithm.
Otherwise, set $t=t+1$ and go to Step 2:

$$
\left\|\mathbf{P}^{t} \overline{\mathbf{X}}^{t}-\mathbf{P}^{t-1} \overline{\mathbf{X}}^{t-1}\right\|<\varepsilon_{2}
$$

where $\varepsilon_{2}>0$ is a small scalar.

Applying Algorithm 5 to solve conditions (11) and (12), the design process for the proposed fuzzy controller design method can be referred to the flowchart described in Figure 1. By finding variables of $\overline{\mathbf{X}}, \mathbf{P}$, and $\mathbf{K}_{i}$ via the design process described in Figure 1, the PDC-based fuzzy controller can be obtained to achieve individual state variance constraint and passivity constraint for the nonlinear discrete-time T-S fuzzy models with multiplicative noise. In the following section, a fuzzy controller design problem for the nonlinear discrete ship steering systems is studied to demonstrate the effectiveness and applicability of proposed multiple constrained fuzzy control approach. 


\section{Fuzzy Controller Design for Nonlinear Discrete Ship Steering Systems}

In this section, the fuzzy control problem for a nonlinear discrete ship steering system is studied to illustrate the applicability of proposed multiple constrained fuzzy controller design method. Referring to [34], the Norrbin nonlinear ship steering equations of motion can be described as follows:

$$
T \ddot{\psi}+\alpha_{1} \dot{\psi}+\beta_{1} v_{1} \dot{\psi}+\beta_{2} v_{2} \dot{\psi}+\alpha_{3} \dot{\psi}^{3}=K \delta+\omega,
$$

where $\psi$ is yaw angle, $r$ is angular velocity, $u$ is rudder angle, and $v$ is noise. Selecting $x_{1}(t)=\psi, x_{2}(t)=\dot{\psi}=r, u(t)=\delta$, $v_{1}(t)=v_{1}, v_{2}(t)=v_{2}$, and $w(t)=\omega$, one has

$$
\begin{gathered}
\dot{x}_{1}(t)=x_{2}(t), \\
\dot{x}_{2}(t)=-\frac{\alpha_{1}}{T} x_{2}(t)-\frac{\alpha_{3}}{T} x_{2}^{3}(t)-\frac{\beta_{1}}{T} x_{2}(t) v_{1}(t) \\
-\frac{\beta_{2}}{T} x_{2}(t) v_{2}(t)+\frac{K}{T} u(t)+\frac{1}{T} w(t) .
\end{gathered}
$$

Applying discretizing technique, it is obvious that the state equations (29a) and (29b) imply

$$
\begin{gathered}
\frac{x_{1}(t+\Delta t)-x_{1}(t)}{\Delta t}=x_{2}(t), \\
\frac{x_{2}(t+\Delta t)-x_{2}(t)}{\Delta t}=-\frac{\alpha_{1}}{T} x_{2}(t)-\frac{\alpha_{3}}{T} x_{2}^{3}(t)-\frac{\beta_{1}}{T} x_{2}(t) v_{1}(t) \\
-\frac{\beta_{2}}{T} x_{2}(t) v_{2}(t)+\frac{K}{T} u(t)+\frac{1}{T} w(t) .
\end{gathered}
$$

It is assumed that the sampling time $\Delta t=0.2$; then one has

$$
\begin{aligned}
& x_{1}(k+1)=x_{1}(k)+x_{2}(k) \Delta t, \\
& x_{2}(k+1) \\
& =\left(1-\frac{\alpha_{1} \Delta t}{T}\right) x_{2}(k)-\frac{\alpha_{3} \Delta t}{T} x_{2}^{3}(k)-\frac{\beta_{1} \Delta t}{T} x_{2}(k) v_{1}(k) \\
& \quad-\frac{\beta_{2} \Delta t}{T} x_{2}(k) v_{2}(k)+\frac{K \Delta t}{T} u(k)+\frac{\Delta t}{T} w(k) .
\end{aligned}
$$

By referring to reference [37], the parameters of the nonlinear ship steering system are represented as $T=261.73 \mathrm{~s}$, $K=0.42 \mathrm{~s}^{-1}, \alpha_{1}=1$, and $\alpha_{3}=30$. Considering the nonlinear discrete ship steering system (31a) and (31b), its corresponding T-S fuzzy model can be described as follows with three fuzzy rules.

Rule 1:

$$
\begin{aligned}
& \text { IF } x_{2}(k) \text { is about } 0 \\
& \text { THEN } x(k+1)=\left[\mathbf{A}_{1}+\sum_{e=1}^{2} \mathbf{N}_{e 1} v_{e 1}(k)\right] \\
& \quad \times x(k)+\mathbf{B}_{1} u(k)+\mathbf{D}_{1} w(k) \\
& y(k)=\mathbf{C}_{1} x(k)+\mathbf{H}_{1} w(k) .
\end{aligned}
$$

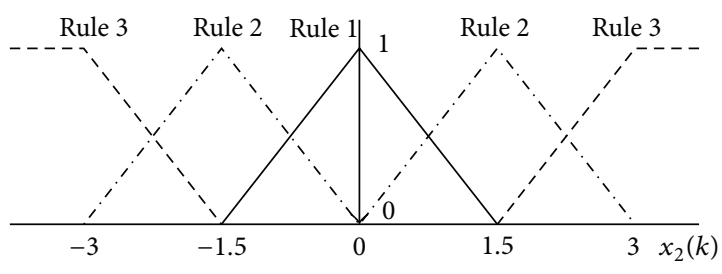

FIgURE 2: The membership functions of $x_{2}(k)$.

Rule 2:

IF $x_{2}(k)$ is about \pm 1.5

$$
\begin{gathered}
\text { THEN } x(k+1)=\left[\mathbf{A}_{2}+\sum_{e=1}^{2} \mathbf{N}_{e 2} v_{e 2}(k)\right] \\
\quad \times x(k)+\mathbf{B}_{2} u(k)+\mathbf{D}_{2} w(k) \\
y(k)=\mathbf{C}_{2} x(k)+\mathbf{H}_{2} w(k) .
\end{gathered}
$$

Rule 3:

IF $x_{2}(k)$ is about \pm 3

$$
\begin{gathered}
\text { THEN } x(k+1)=\left[\mathbf{A}_{3}+\sum_{e=1}^{2} \mathbf{N}_{e 3} v_{e 3}(k)\right] \\
\times x(k)+\mathbf{B}_{3} u(k)+\mathbf{D}_{3} w(k) \\
y(k)=\mathbf{C}_{3} x(k)+\mathbf{H}_{3} w(k),
\end{gathered}
$$

where

$$
\begin{gathered}
\mathbf{A}_{1}=\left[\begin{array}{cc}
1 & 0.2 \\
0 & 0.999
\end{array}\right], \quad \mathbf{A}_{2}=\left[\begin{array}{cc}
1 & 0.2 \\
0 & 0.948
\end{array}\right], \\
\mathbf{A}_{3}=\left[\begin{array}{cc}
1 & 0.2 \\
0 & 0.793
\end{array}\right], \\
\mathbf{N}_{11}=\mathbf{N}_{12}=\mathbf{N}_{13}=\left[\begin{array}{cc}
0 & 0 \\
0 & 0.00023
\end{array}\right], \\
\mathbf{N}_{21}=\mathbf{N}_{22}=\mathbf{N}_{23}=\left[\begin{array}{ll}
0 & 0 \\
0 & 0.00031
\end{array}\right], \\
\mathbf{B}_{1}=\mathbf{B}_{2}=\mathbf{B}_{3}=\left[\begin{array}{cc}
0 \\
0.00032
\end{array}\right] \\
\mathbf{D}_{1}=\mathbf{D}_{2}=\left[\begin{array}{c}
0 \\
0.00076
\end{array}\right] \\
\mathbf{C}_{1}=\mathbf{C}_{2}=\left[\begin{array}{ll}
0 & 1
\end{array}\right] \\
\mathbf{H}_{1}=\mathbf{H}_{2}=1 .
\end{gathered}
$$

The membership function for the state $x_{2}(k)$ is given in Figure 2.

Employing the PDC technique, the corresponding fuzzy controller can be constructed as follows.

Rule 1:

$$
\begin{aligned}
& \text { IF } x_{2}(k) \text { is about } 0 \\
& \text { THEN } u(k)=\mathbf{F}_{1} x(k) .
\end{aligned}
$$


Rule 2:

$$
\begin{aligned}
& \text { IF } x_{2}(k) \text { is about } \pm 1.5 \\
& \text { THEN } u(k)=\mathbf{F}_{2} x(k) .
\end{aligned}
$$

Rule 3:

$$
\begin{aligned}
& \text { IF } x_{2}(k) \text { is about } \pm 3 \\
& \text { THEN } u(k)=\mathbf{F}_{3} x(k) \text {. }
\end{aligned}
$$

For starting analyzing and designing, let us select the supply rate $\gamma=0.6, \mathbf{S}=\mathbf{I}$ with compatible dimension and assign the variance constraints as $\sigma_{1}^{2}=0.2, \sigma_{2}^{2}=0.1$. Solving conditions (11) and (12) of Theorem 3 by Algorithm 5, the matrix $\overline{\mathbf{X}}$ and $\mathbf{F}_{i}$ can be obtained as follows:

$$
\begin{aligned}
& \overline{\mathbf{X}}=\left[\begin{array}{cc}
0.0442 & -0.0042 \\
-0.0042 & 0.0372
\end{array}\right], \\
& \mathbf{F}_{1}=\left[\begin{array}{ll}
-289.2 & -3174.1
\end{array}\right], \\
& \mathbf{F}_{2}=\left[\begin{array}{ll}
-289.2 & -3014.7
\end{array}\right], \\
& \mathbf{F}_{3}=\left[\begin{array}{ll}
-289.2 & -2530.3
\end{array}\right]
\end{aligned}
$$

In the simulations, the external disturbances are all chosen as zero-mean white noises with variance one. The final responses of states are shown in Figures 3 and 4 with initial condition $x(0)=\left[\begin{array}{ll}1 & 0\end{array}\right]^{T}$. From the simulation results, the effect of the external disturbance $w(t)$ on the controlled system can be criticized as follows:

$$
\frac{E\left\{2 \sum_{k=0}^{k_{q}} y^{T}(k) w(k)\right\}}{E\left\{\sum_{k=0}^{k_{q}} w^{T}(k) w(k)\right\}}=1.995 .
$$

The ratio value of (41) is bigger than determined dissipation rate $\gamma=0.6$; one can find that condition (6) of Definition 1 is satisfied. Besides, the variances of states $x_{1}(t)$ and $x_{2}(t)$ are 0.0477 and $3.3144 \times 10^{-4}$, respectively. It is obvious that the individual state variance constraints are all satisfied.

\section{Conclusions}

In this paper, a multiple constrained fuzzy control methodology has been developed for nonlinear discrete ship steering stochastic systems. Applying T-S fuzzy model to represent the nonlinear discrete ship steering stochastic system, a PDCbased fuzzy controller was developed to achieve passivity constraint and individual state variance constraint. The sufficient conditions derived in this paper are nonstrict LMI conditions that cannot be solved by numerical convex optimal programming algorithm. Thus, a modified ILMI algorithm was provided in this paper to solve the proposed nonlinear matrix inequality conditions. From the control simulations of nonlinear discrete ship steering stochastic systems, one can find that the proposed fuzzy controller design methodology can provide an effective scheme to control nonlinear discrete stochastic systems subject to passivity constraint and individual state variance constraint, simultaneously.

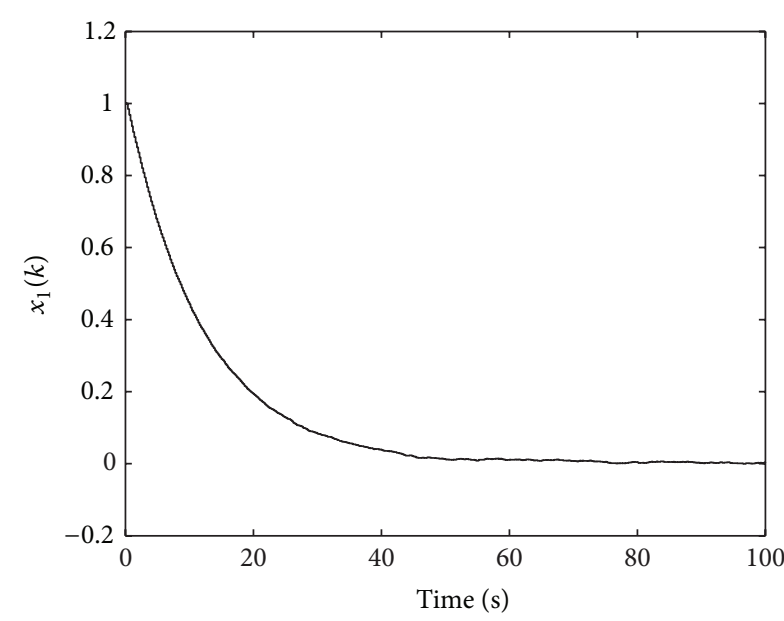

FIGURE 3: Responses of state $x_{1}(k)$.

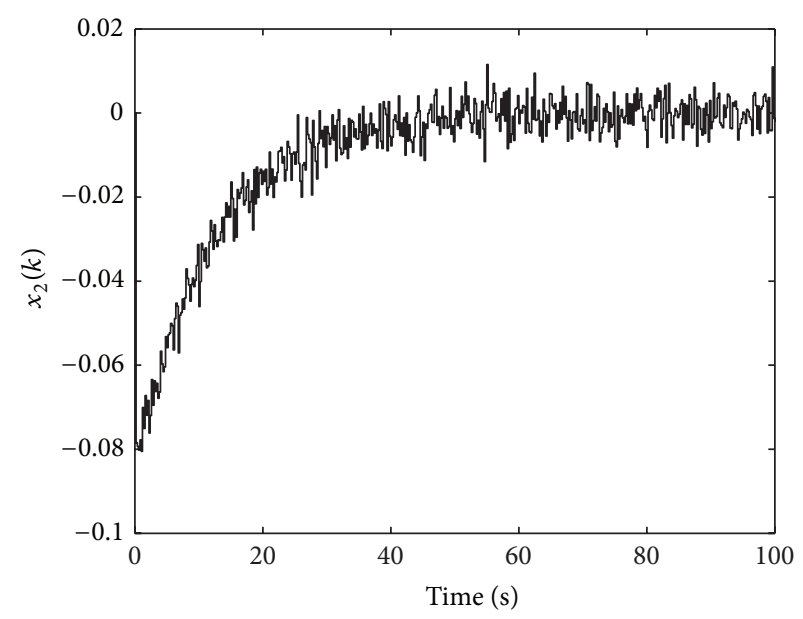

FIGURE 4: Responses of state $x_{2}(k)$.

\section{Conflict of Interests}

The authors declare that there is no conflict of interests regarding the publication of this paper.

\section{Acknowledgments}

The authors would like to express their sincere gratitude to anonymous reviewers who gave them some constructive comments, criticisms, and suggestions. This work was supported by the National Science Council of the Republic of China under Contract NSC102-2221-E-019-051.

\section{References}

[1] W.-J. Chang and H.-Y. Chung, "A covariance controller design incorporating optimal estimation for nonlinear stochastic systems," Journal of Dynamic Systems, Measurement and Control, Transactions of the ASME, vol. 118, no. 2, pp. 346-349, 1996. 
[2] K.-Y. Chang, W.-J. Wang, and W.-J. Chang, "Covariance control for stochastic multivariable systems with hysteresis nonlinearity," International Journal of Systems Science, vol. 28, no. 7, pp. 731-736, 1997.

[3] D. Yue and Q.-L. Han, "Delay-dependent exponential stability of stochastic systems with time-varying delay, nonlinearity, and Markovian switching," IEEE Transactions on Automatic Control, vol. 50, no. 2, pp. 217-222, 2005.

[4] Z. Wang, Y. Liu, and X. Liu, " $\mathrm{H}_{\infty}$ filtering for uncertain stochastic time-delay systems with sector-bounded nonlinearities," Automatica, vol. 44, no. 5, pp. 1268-1277, 2008.

[5] H. Shen, S. Y. Xu, J. P. Zhou, and J. J. Lu, "Fuzzy $\mathrm{H}_{\infty}$ filtering for nonlinear Markovian jump neutral systems," International Journal of Systems Science, vol. 42, no. 5, pp. 767-780, 2011.

[6] E. E. Yaz and Y. I. Yaz, "State estimation of uncertain nonlinear stochastic systems with general criteria," Applied Mathematics Letters, vol. 14, no. 5, pp. 605-610, 2001.

[7] L. Ma, Y. Bo, Y. Zhou, and Z. Guo, "Error variance-constrained $\mathrm{H}_{\infty}$ filtering for a class of nonlinear stochastic systems with degraded measurements: the finite horizon case," International Journal of Systems Science, vol. 43, no. 12, pp. 2361-2372, 2012.

[8] W. Lin, "Mixed $\mathrm{H}_{2} / \mathrm{H}_{\infty}$ control of nonlinear systems," in Proceedings of the 34th IEEE Conference on Decision and Control, pp. 333-338, December 1995.

[9] F. Yang, Z. Wang, and D. W. C. Ho, "Robust mixed $\mathrm{H}_{2} / \mathrm{H}_{\infty}$ control for a class of nonlinear stochastic systems," IEE Proceedings, vol. 153, no. 2, pp. 175-184, 2006.

[10] B.-S. Chen, C.-S. Tseng, and H.-J. Uang, "Mixed $\mathrm{H}_{2} / \mathrm{H}_{\infty}$ fuzzy output feedback control design for nonlinear dynamic systems: an LMI approach," IEEE Transactions on Fuzzy Systems, vol. 8, no. 3, pp. 249-265, 2000.

[11] A. Hotz and R. E. Skelton, "A covariance control theory," International Journal of Control, vol. 46, no. 1, pp. 13-32, 1987.

[12] C. Hsieh and R. E. Skelton, "All covariance controllers for linear discrete-time systems," IEEE Transactions on Automatic Control, vol. 35, no. 8, pp. 908-915, 1990.

[13] R. E. Skelton, T. Iwasaki, and K.M. Grigoriadis, A Unified Approach to Linear Control Design, Taylor \& Francis, London, UK, 1997.

[14] W. J. Chang and S. M. Wu, "Covariance control for fuzzy-based nonlinear stochastic systems," International Journal of Fuzzy Systems, vol. 5, no. 4, pp. 221-228, 2003.

[15] W.-J. Chang and C.-C. Shing, "Discrete fuzzy covariance control for specified decay rate," International Journal of Systems Science, vol. 36, no. 4, pp. 201-208, 2005.

[16] S. Xie, L. Xie, and C. E. de Souxa, "Robust dissipative control for linear systems with dissipative uncertainty," International Journal of Control, vol. 70, no. 2, pp. 169-191, 1998.

[17] Z. Tan, Y. C. Soh, and L. Xie, "Dissipative control for linear discrete-time systems," Automatica, vol. 35, no. 9, pp. 1557-1564, 1999.

[18] Z. Li, J. Wang, and H. Shao, "Delay-dependent dissipative control for linear time-delay systems," Journal of the Franklin Institute, vol. 339, no. 6-7, pp. 529-542, 2002.

[19] C. Li, H. Zhang, and X. Liao, "Passivity and passification of fuzzy systems with time delays," Computers and Mathematics with Applications, vol. 52, no. 6-7, pp. 1067-1078, 2006.
[20] X.-Z. Dong, "Robust strictly dissipative control for discrete singular systems," IET Control Theory and Applications, vol. 1, no. 4, pp. 1060-1067, 2007.

[21] W.-J. Chang, L.-Z. Liu, and C.-C. Ku, "Passive fuzzy controller design via observer feedback for stochastic Takagi-Sugeno fuzzy models with multiplicative noises," International Journal of Control, Automation and Systems, vol. 9, no. 3, pp. 550-557, 2011.

[22] W.-J. Chang, S.-S. Jheng, and C.-C. Ku, "Fuzzy control with robust and passive properties for discrete-time Takagi-Sugeno fuzzy systems with multiplicative noises," Proceedings of the Institution of Mechanical Engineers. Part I: Journal of Systems and Control Engineering, vol. 226, no. 4, pp. 476-485, 2012.

[23] H. Shen, S. Y. Xu, J. W. Lu, and J. Zhou, "Passivity-based control for uncertain stochastic jumping systems with mode-dependent round-trip time delays," Journal of the Franklin Institute, vol. 349, no. 5, pp. 1665-1680, 2012.

[24] Z. G. Wu, J. Lam, H. Y. Su, and J. Chu, "Stability and dissipativity analysis of static neural networks with time delay," IEEE Transactions on Neural Networks and Learning Systems, vol. 23, no. 2, pp. 199-210, 2012.

[25] Z. G. Wu, P. Shi, H. Y. Su, and J. Chu, "Dissipativity analysis for discrete-time stochastic neural networks with time-varying delays," IEEE Transactions on Neural Networks and Learning Systems, vol. 24, no. 3, pp. 345-355, 2013.

[26] H. Zhang, Y. Shi, and A. S. Mehr, "On $\mathrm{H}_{\infty}$ filtering for discretetime takagi-sugeno fuzzy systems," IEEE Transactions on Fuzzy Systems, vol. 20, no. 2, pp. 396-401, 2012.

[27] H. Zhang, Y. Shi, and B. X. Mu, "Optimal $\mathrm{H}_{\infty}$-based linearquadratic regulator tracking control for discrete-time TakagiSugeno fuzzy systems with preview actions," Journal of Dynamic Systems, Measurement, and Control, vol. 135, no. 4, 2013.

[28] H. Zhang, M. X. Liu, Y. Shi, and J. Sheng, "Extended LMI representatives for stability and stabilization of discrete-time Takagi-Sugeno fuzzy systems," Optimal Control Applications and Methods, 2013.

[29] S. He and F. Liu, "Controlling uncertain fuzzy neutral dynamic systems with Markov jumps," Journal of Systems Engineering and Electronics, vol. 21, no. 3, pp. 476-484, 2010.

[30] S. He and F. Liu, "Robust finite-time stabilization of uncertain fuzzy jump systems," International Journal of Innovative Computing, Information and Control, vol. 6, no. 9, pp. 3853-3862, 2010.

[31] E. Kim and S. Kim, "Stability analysis and synthesis for an affine fuzzy control system via LMI and ILMI: continuous case," IEEE Transactions on Fuzzy Systems, vol. 10, no. 3, pp. 391-400, 2002.

[32] W. J. Chang, Y. T. Meng, and K. H. Tsai, "AQM router design for TCP network via input constrained fuzzy control of timedelay affine Takagi-Sugeno fuzzy models," International Journal of Systems Science, vol. 43, no. 12, pp. 2297-2313, 2012.

[33] J.-W. Dong and S.-T. Zhang, "Design of ship motion controller based on discrete fuzzy multi-model approach," in Proceedings of the 4th IEEE Conference on Industrial Electronics and Applications (ICIEA '09), pp. 1-5, May 2009.

[34] W. J. Chang, M. W. Chen, and C. C. Ku, "Passive fuzzy controller design for discrete ship steering systems via Takagi-Sugeno fuzzy model with multiplicative noises," Journal of Marine Science and Technology, vol. 21, no. 2, pp. 159-165, 2013. 
[35] H.-Y. Chung and W.-J. Chang, "Input and state covariance control for bilinear stochastic discrete systems," Control, Theory and Advanced Technology, vol. 6, no. 4, pp. 655-667, 1990.

[36] S. Boyd, L. E. Ghaoui, E. Feron, and V. Balakrishnan, Linear Matrix Inequalities in System and Control Theory, SIAM, Philadelphia, Pa, USA, 1994.

[37] S.-T. Zhang and S.-J. Wang, "Design of discrete fuzzy controller for Norrbin ship steering," in Proceedings of the Chinese Control and Decision Conference (CCDC '08), pp. 2599-2603, July 2008. 


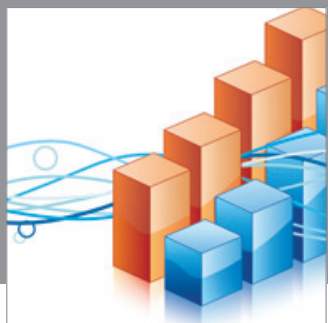

Advances in

Operations Research

mansans

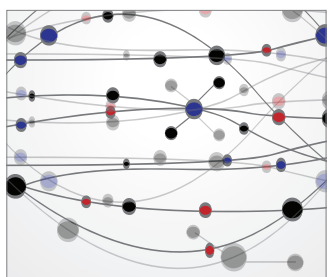

The Scientific World Journal
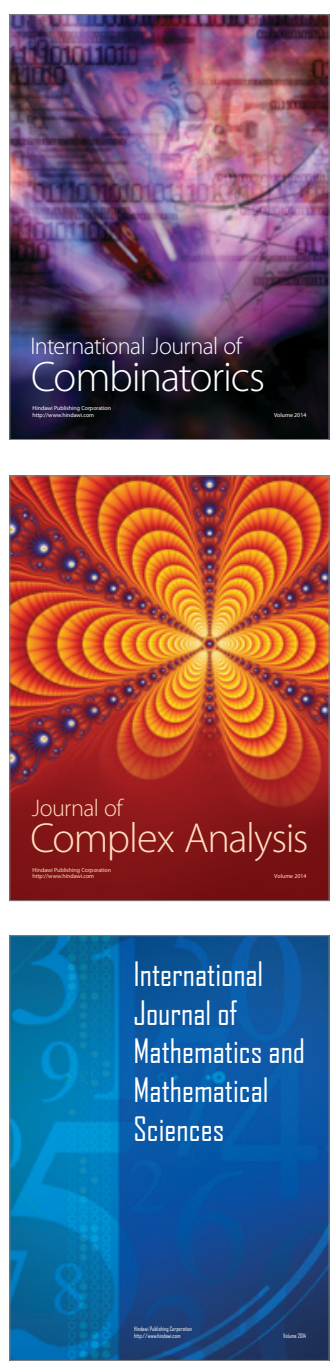
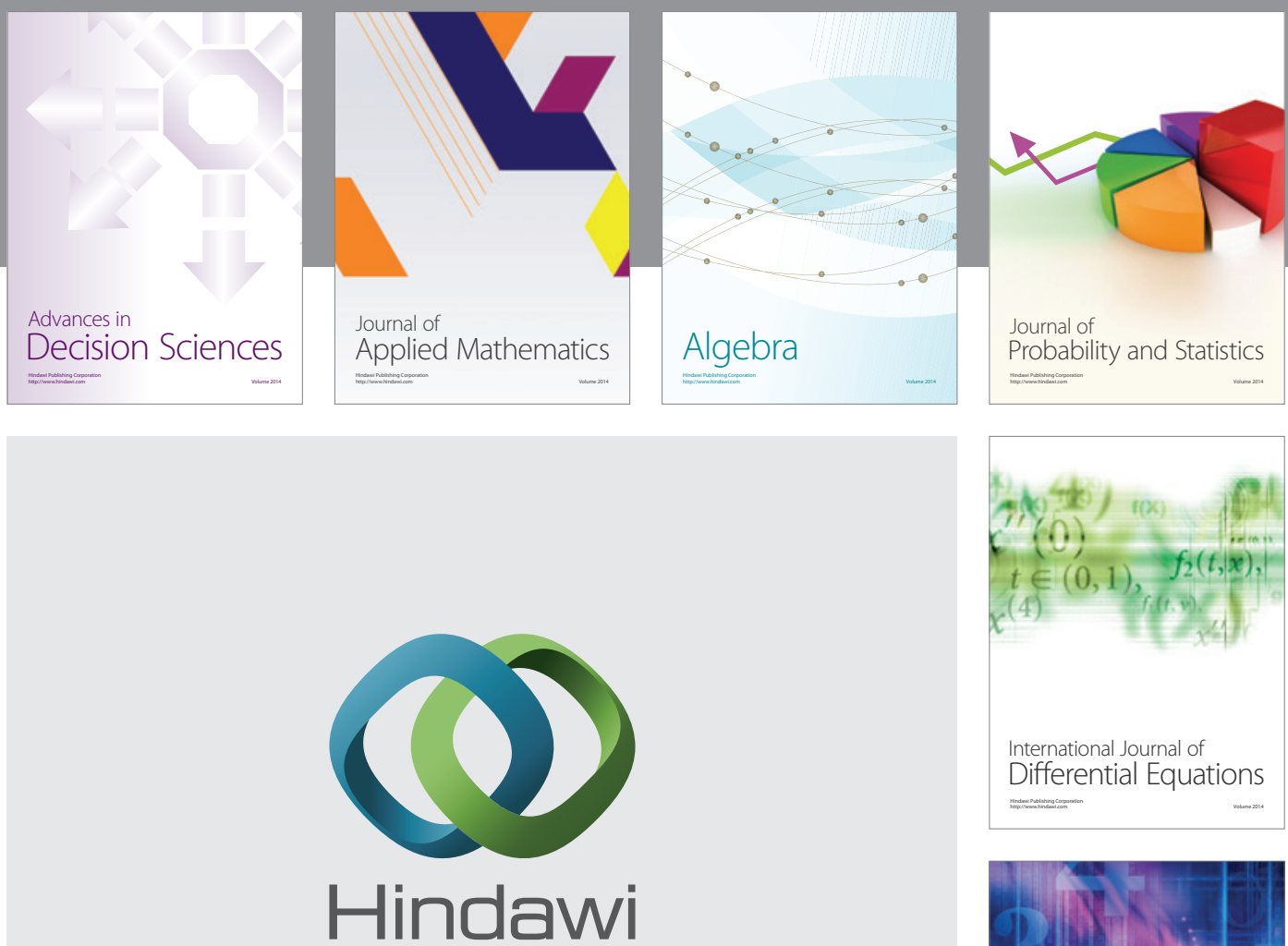

Submit your manuscripts at http://www.hindawi.com
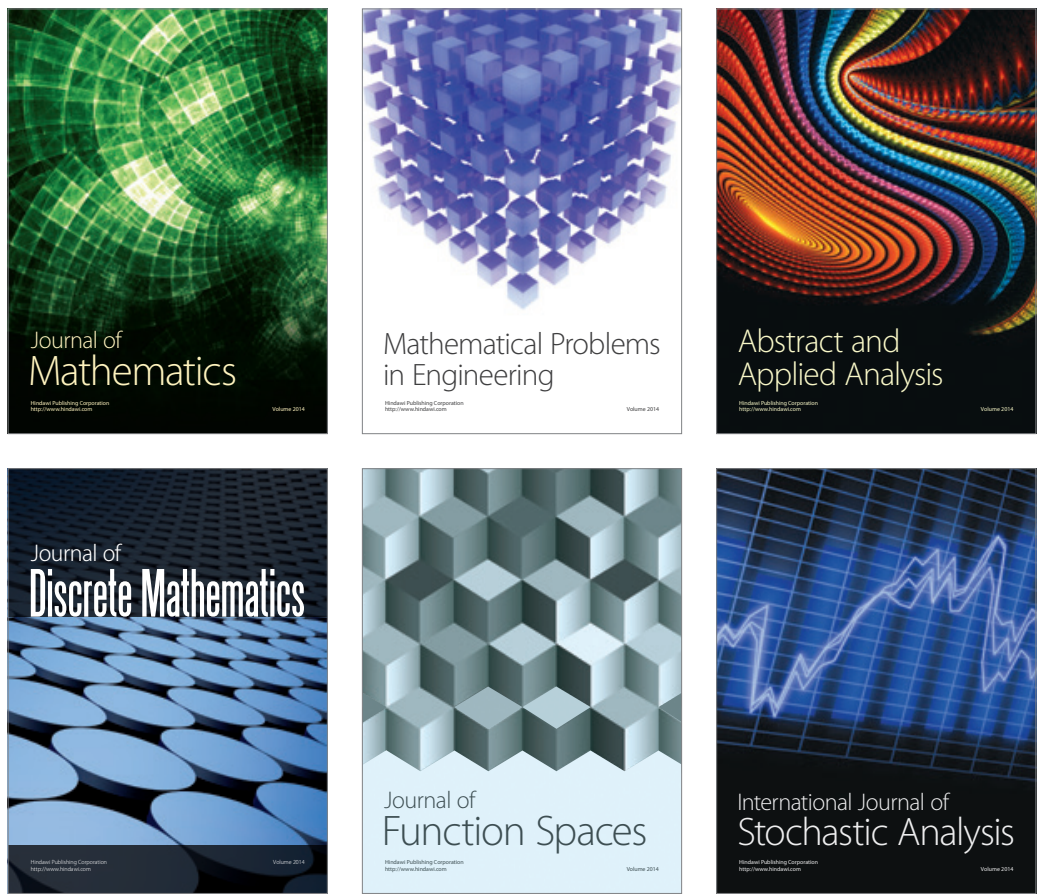

Journal of

Function Spaces

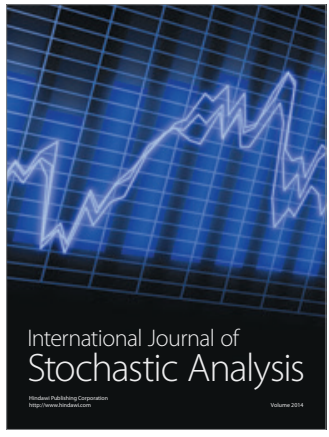

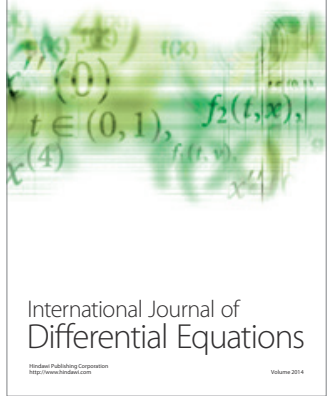
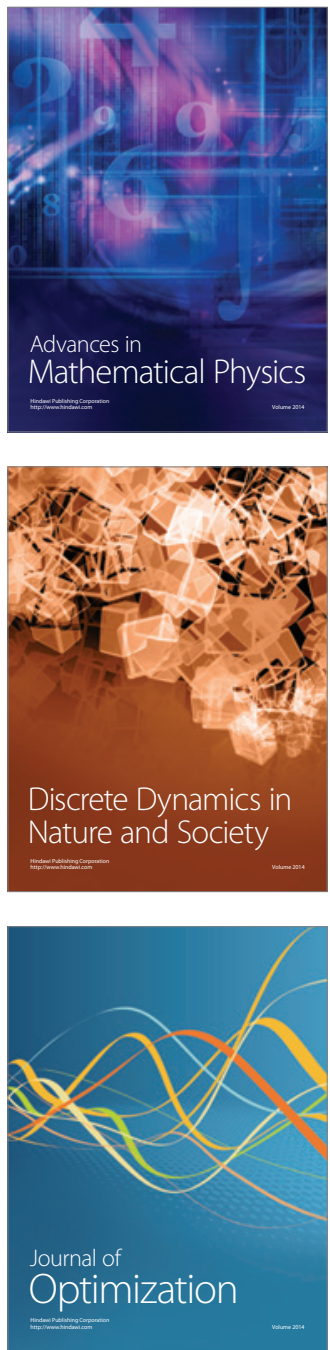